\title{
Plant-Made Bet v 1 for Molecular Diagnosis
}

\author{
Mattia Santoni ${ }^{1}$, Maria Antonietta Ciardiello ${ }^{2}$, Roberta Zampieri ${ }^{1}$, Mario Pezzotti ${ }^{1}$, \\ Ivana Giangrieco ${ }^{2,3}$, Chiara Rafaiani ${ }^{4}$, Michela Ciancamerla ${ }^{4}$, Adriano Mari ${ }^{3,4 *}$ \\ and Linda Avesani ${ }^{*}$ *
}

${ }^{1}$ Department of Biotechnology, University of Verona, Verona, Italy, ${ }^{2}$ Institute of Bioscience and BioResources, CNR, Naples, Italy, ${ }^{3} \mathrm{ADL}$ (Allergy Data Laboratories) S.r.l., Latina, Italy, ${ }^{4}$ Associated Centre for Molecular Allergology, Rome, Italy

\section{OPEN ACCESS}

Edited by:

Jussi Joonas Joensuu,

VTT Technical Research Centre of Finland Ltd, Finland

Reviewed by: Mario Sergio Palma, Paulista State University Júlio de Mesquita Filho Rio Claro, Brazil

Anna Gieras,

University Medical Center Hamburg-Eppendorf, Germany

${ }^{*}$ Correspondence: Adriano Mari adriano.mari@caam-allergy.com Linda Avesan

linda.avesani@univr.it

Specialty section: This article was submitted to Plant Metabolism and Chemodiversity, a section of the journal Frontiers in Plant Science

Received: 04 February 2019 Accepted: 12 September 2019

Published: 10 October 2019

Citation:

Santoni M, Ciardiello MA, Zampieri R, Pezzotti M, Giangrieco I, Rafaiani C, Ciancamerla M, Mari A and Avesani L (2019) Plant-Made Bet $v 1$ for Molecular Diagnosis.

Front. Plant Sci. 10:1273. doi: 10.3389/fp/s.2019.01273
Allergic disease diagnosis is currently experiencing a breakthrough due to the use of allergenic molecules in serum-based assays rather than allergen extracts in skin tests. The former methodology is considered a very innovative technology compared with the latter, since it is characterized by flexibility and adaptability to the patient's clinical history and to microtechnology, allowing multiplex analysis. Molecular-based analysis requires pure allergens to detect IgE sensitization, and a major goal, to maintain the diagnosis costeffective, is to limit their production costs. In addition, for the production of recombinant eukaryotic proteins similar to natural ones, plant-based protein production is preferred to bacterial-based systems due to its ability to perform most of the post-translational modifications of eukaryotic molecules. In this framework, Plant Molecular Farming (PMF) may be useful, being a production platform able to produce complex recombinant proteins in short time-frames at low cost. As a proof of concept, PMF has been exploited for the production of Bet v 1a, a major allergen associated with birch (Betula verrucosa) pollen allergy. Bet $v$ 1a has been produced using two different transient expression systems in Nicotiana benthamiana plants, purified and used in a new generation multiplex allergy diagnosis system, the patient-Friendly Allergen nano-BEad Array (FABER). Plant-made Bet $v 1 \mathrm{a}$ is immunoreactive, binding lgE and inhibiting lgE-binding to the Escherichia coli expressed allergen currently available in the FABER test, thus suggesting an overall similar though non-overlapping immune activity compared with the $E$. coli expressed form.

Keywords: allergen, molecular farming, transient expression, IgE, structure homology

\section{INTRODUCTION}

Allergic diseases, defined as abnormal responses of the human body when in contact with an allergen, have become a common health problem worldwide, with a rising incidence both in adults and children (Lau et al., 2018). It is rather difficult to obtain exact epidemiology data on this topic but it has been estimated that $25 \%$ of the general population suffer from these diseases (Sampson, 2004).

The primary diagnostic tool currently used for allergic disease diagnosis is skin prick testing (SPT), which evaluates the presence and degree of cutaneous reactivity against a surrogate marker of sensitization, composed of protein extracts from allergenic sources. However, this method is hampered by several problems. For instance, it cannot be used in patients who have extensive eczema, dermographism or urticaria or who are taking antihistamines and/or other medications. 
More importantly, it frequently fails to detect specific IgE because the composition of the surrogate markers can be very variable (Giangrieco et al., 2012). This high variability makes it impossible to have standardized extracts with a constant allergen composition, since this may be influenced by several factors, such as differences among plant cultivars, post-harvest treatments, specific phenological stages, and extraction protocols (Ciardiello et al., 2009; Pasquariello et al., 2012). Molecule-based diagnosis may overcome all these drawbacks, given the possibility of detecting the specific IgE against individual allergenic molecules (Giangrieco et al., 2012; Ciardiello et al., 2013).

Molecular-based diagnosis, furthermore, may be used to predict biopharmaceuticals with a potential use in therapy for tolerance induction to allergens in a drug-companion diagnostic strategy.

The allergens used for molecular diagnosis may be extracted from the original sources or may be produced recombinantly in different platforms. A plant-based platform, in this framework, represents both a cost-effective and speedy system for producing allergens and, in particular, for the expression of plant allergens. In fact, it provides an environment useful to obtain features, such as post-translational modifications and protein folding similar to those present in the molecules of the authentic source.

In this study, we have transiently transformed Nicotiana benthamiana plants for the production of one of the major allergens associated with birch pollen allergy, Bet v 1a (UniProtKB accession number P15494) (Radauer et al., 2008). Bet $\mathrm{v}$ 1a is a $17-\mathrm{kDa}$ protein which shares epitopes with the major pollen allergens of trees belonging to the Fagales order and with some plant-derived foods (Niederberger et al., 1998). Bet v l represents a target for IgE antibodies of more than $95 \%$ of patients allergic to birch pollen, and almost $60 \%$ of them are exclusively sensitized to Bet v 1 (Jarolim et al., 1989).

Here we report the setup of a plant-based system for allergen production that was tested with the expression of recombinant Bet v 1a. For this purpose, two different transient systems in terms of yield and timeframe for protein upstream processing were used, and the results were compared. In addition, the features of the recombinant product were characterized. In particular, the folding of the plant-made Bet v 1a (pBet v 1a) was investigated by circular dichroism measurements, whereas the evaluation of its immunological reactivity (IgE binding) was analyzed with the FABER multiplex system by direct testing and experiments of IgE binding inhibition.

\section{MATERIALS AND METHODS}

\section{Vectors and Plant Transformation}

The DNA sequence encoding the allergen Bet $\mathrm{v}$ 1a was designed with the following modifications: the codon usage was optimized for $N$. benthamiana and a poly-Histidine tag, a Flag-tag, and a linker (GPGP) were added at the N-terminus. The synthetic gene (Invitrogen GeneArt Gene Synthesis) was then inserted into the $\mathrm{pENTR}^{\mathrm{TM}} / \mathrm{D}-\mathrm{TOPO}$ vector, following the manufacturer's instructions, and sequenced to assess the absence of errors. The resulting vector was recombined by Gateway ${ }^{\mathrm{TM}}$ LR Clonase ${ }^{\mathrm{TM}}$ II Enzyme mix (ThermoFisher) in the two destination vectors pK7WG2 (Karimi et al., 2002) and pG PVX GATEWAY(A) (Avesani et al., 2007).

The final result consisted of two vectors, pK7WG2.Betv1 and pGPVXGATEWAY(A).Betv1, that were inserted into the Agrobacterium tumefaciens, EHA105 and GV3101 strains, respectively, by electroporation.

\section{pBet v 1a Transient Expression in N. Benthamiana}

Nicotiana benthamiana plants were grown from seeds and cultivated in a growth chamber at $25^{\circ} \mathrm{C}$ with a light/dark cycle of $16 \mathrm{~h} / 8 \mathrm{~h}$ and a relative humidity of $20 \%$ to $40 \%$.

A. tumefaciens cells, both EHA105 and GV3101, carrying pK7WG2.Betv1 and pGPVXGATEWAY(A).Betv1 were seeded into a lysogeny broth (LB) medium containing $50 \mu \mathrm{g} / \mathrm{ml}$ of rifampicin, $300 \mu \mathrm{g} / \mathrm{ml}$ of streptomycin, and $100 \mu \mathrm{g} / \mathrm{ml}$ of spectomycin for pK7WG2.Betv1 or $50 \mu \mathrm{g} / \mathrm{ml}$ of rifampicin, $50 \mu \mathrm{g} /$ $\mathrm{ml}$ of kanamycin, and $5 \mu \mathrm{g} / \mathrm{ml}$ of tetracyclin for pGPVXGAT(A). Betv1. Empty vectors were used as negative controls. For syringe agroinfiltration, performed as described in Gecchele et al. (2015), overnight bacterial cultures were collected by centrifugation at $4500 \mathrm{~g}$, re-suspended in the infiltration buffer $(10 \mathrm{mM} \mathrm{MES} \mathrm{pH}$ $5.5,10 \mathrm{mM} \mathrm{MgSO} 4$, and $100 \mu \mathrm{M}$ acetosyringone) at an optical density of 0.8 at $600 \mathrm{~nm}$. Following a 3-h incubation, the culture was used for the syringe infiltratation of 4- to 5-week-old N. benthamiana plants.

After the infiltration, the plants carrying the pK7WG2. Betv1 vector were sampled from the third day post-inoculation (dpi) to the 14th dpi; the plants infiltrated with the pG PVX GATEWAY(A).Betv1 were harvested after the symptom appearance between 10 to $14 \mathrm{dpi}$.

\section{pBet v 1a Detection}

Total soluble proteins (TSP) were extracted from the leaves by grinding the tissue sample to a fine powder under liquid nitrogen. The powder was re-suspended in three volumes of extraction buffer ( $1 \times$ phosphate-buffered saline [PBS], 0.1\% Tween-20) supplemented with cOmplete ${ }^{\mathrm{TM}}$ EDTA-free protease inhibitor (COEDTAF-RO).

The homogenate was centrifuged at $30,000 \mathrm{~g}$ for $20 \mathrm{~min}$ at $4^{\circ} \mathrm{C}$. The protein concentration was determined using the Bradford reagent (Sigma B6916).

The presence of pBet $\mathrm{v} 1 \mathrm{a}$ in the homogenate was detected by Western blot analysis. Briefly, equal quantities of TSP were loaded onto a $14 \%$ reducing SDS-PAGE. After the electrophoretic separation, the proteins were transferred onto a nitrocellulose membrane by electroblotting and incubated with anti-polyHisitidine and anti-FLAG ${ }^{\circledR}$ antibodies, diluted 1:5000 and 1:1000, respectively. The protein band recognized by the antibodies was detected using the $\mathrm{ECL}^{\mathrm{TM}}$ Select Western Blotting Detection Reagent (Amersham). In particular, the chemiluminescent signal was captured with the Chemidoc $^{\mathrm{TM}}$ (BioRad). 


\section{pBet v 1a Immobilized Metal Affinity Chromatography (IMAC) Purification}

The purification of the allergen was carried out as described in Bortesi et al. (2009). Briefly, 10 to $30 \mathrm{~g}$ of leaf tissue were homogenized in four volumes of buffer $(1 \times$ PBS, $10 \mathrm{mM}$ ascorbic acid, $0.1 \%$ Tween-20, pH 6.0). To remove the insoluble material, the homogenate was centrifuged at $15,000 \mathrm{~g}$, at $4^{\circ} \mathrm{C}$ for $15 \mathrm{~min}$. Next, the supernatant was removed, passed through a filter paper and adjusted to $500 \mathrm{mM} \mathrm{NaCl}, 5 \mathrm{mM}$ imidazole, $\mathrm{pH}$ 8. After incubation with gentle shaking for $1 \mathrm{~h}$ in ice, a last centrifugation step at $4^{\circ} \mathrm{C}$ for $30 \mathrm{~min}$ at $30,000 \mathrm{~g}$ produced a clear supernatant. To remove non-specifically bound material, the supernatant was loaded onto a disposable column packed with $1 \mathrm{ml} \mathrm{Ni-NTA} \mathrm{resin}$ (QIAGEN), using a gravity flow. The column was washed with a PBS solution containing $500 \mathrm{mM} \mathrm{NaCl}, 0.1 \%$ Tween-20, 10 $\mathrm{mM}$ imidazole, $\mathrm{pH}$ 8. $\mathrm{pBet} \mathrm{v}$ la was eluted with an increasing concentration of imidazole, ranging from 10 to $200 \mathrm{mM}$, using a gradient maker. The purity of the allergen preparation was analyzed by loading aliquots of the eluted fractions on reducing SDS-PAGE, followed by silver staining (Mortz et al., 2001). The pure fractions were pooled and dialyzed against $1 \times$ PBS.

\section{Size Exclusion Chromatography}

The purified Bet $\mathrm{v}$ 1a was subjected to size exclusion chromatography using a fast protein liquid chromatography (FPLC) system, model AKTA pure 25L- Gold seal (GE Healthcare Europe $\mathrm{GmbH}$, Milan, Italy). The purified protein was loaded on a gel filtration column Superdex 75 HR10/30 (Amersham Biosciences, Uppsala, Sweden), equilibrated, and eluted with 10 $\mathrm{mM}$ Tris- $\mathrm{HCl}, \mathrm{pH} 7.5,0.25 \mathrm{M} \mathrm{NaCl}$. Fractions of $0.5 \mathrm{ml}$ were collected, and the absorbance at $280 \mathrm{~nm}$ was recorded.

\section{RP-HPLC Chromatography}

Aliquots of the protein fraction collected from the size exclusion chromatography were subjected to RP-HPLC separation. The protein was loaded on a Vydac (Deerfield, IL, USA) C8 column $(4.6 \times 250 \mathrm{~mm})$, using a Beckman System Gold apparatus (Fullerton, CA, USA). Elution was performed by a multistep linear gradient of eluent $\mathrm{B}(0.08 \%$ TFA in acetonitrile) in eluent $\mathrm{A}$ $(0.1 \% \mathrm{TFA})$ at a flow rate of $1 \mathrm{ml} / \mathrm{min}$. The eluate was monitored at 220 and $280 \mathrm{~nm}$.

\section{Estimation of the Pure Protein Concentration}

The concentration of the pure protein obtained from size exclusion chromatography was estimated on the basis of the molar extinction coefficient, at $280 \mathrm{~nm}\left(11,920 \mathrm{M} \cdot \mathrm{cm}^{-1}\right)$, calculated for pBet v 1a (178 residues) using the ProtParam tool on the Exapsy server (www.expasy.org).

\section{Mass Spectrometry Experiments}

The protein sample $(5 \mu \mathrm{g})$ deriving from the RP-HPLC elution was dried with a centrifugal vacuum concentrator (Savant Speedvac Plus SC110A, Ramsey, Minnesota, USA) and solubilized in ammonium bicarbonate (AMBIC) $0.1 \mathrm{M}$. Next, a standard protocol of reduction, alkylation, and digestion with trypsin was applied. Briefly, the protein sample was reduced with $1 \mathrm{mM}$ dithiothreithol in $100 \mathrm{mM}$ AMBIC and alkylated with $5.5 \mathrm{mM}$ iodoacetamide (IAA) in $10 \%$ acetonitrile and $10 \mathrm{mM}$ AMBIC. The protein was then desalted using ZipTip C18 tips (Millipore, Billerica, MA) and incubated overnight at a trypsin/substrate ratio of $1: 50$ at $37^{\circ} \mathrm{C}$ for $20 \mathrm{~h}$. The obtained peptides were separated using an Ultimate 3000 instrument (LC Packings, Sunnyvale, CA) 2D-nano-HPLC online interfaced with a QSTAR-Elite Hewlett Packard, Series 1100 HPLC with UV detector. The peptide sequence identification was obtained using the Mascot Server (Matrix Science, London, UK) on the website www.matrixscience.com.

\section{Circular Dichroism (CD) Experiments}

CD spectra were recorded on a JASCO J-810 spectropolarimeter (Easton, MD) as already reported (Offermann et al., 2015). A quartz cell of $0.1-\mathrm{cm}$ path length was used to record the spectra over the wavelength range of 260 to $195 \mathrm{~nm}$ with a bandwidth of $1.0 \mathrm{~nm}$ and a time constant of $8.0 \mathrm{~s}$. For the CD experiments, the protein solution was diluted in the appropriate buffer and left for $2 \mathrm{~h}$ at $25^{\circ} \mathrm{C}$ before the acquisition of the spectra. The measurements were performed in PBS containing $0.1 \%$ Tween at $25^{\circ} \mathrm{C}$. The protein concentration was $0.10 \mathrm{mg} / \mathrm{ml}$. Each spectrum was baseline corrected for the contribution of the solvent.

For the thermostability experiments, purified pBet $\mathrm{v}$ 1a $(0.1 \mathrm{mg} / \mathrm{ml})$ was incubated for $5 \mathrm{~min}$ in PBS containing $0.1 \%$ Tween, at different temperatures. After each incubation, the CD spectrum was recorded as described above.

\section{Specific IgE Detection by the FABER Testing System}

FABER (ADL S.r.l., Latina, Italy) is a multiplex in vitro serological test that allows the detection of $\operatorname{IgE}$ antibodies produced by allergic subjects, specifically recognizing the allergens spotted on the biochip (Alessandri et al., 2017; Tuppo et al., 2018). The FABER version used to perform the present study (FABER 244122-122) bears 244 allergenic preparations, representing 122 purified molecular allergens and 122 multiple protein allergenic extracts. The data were obtained using 304 sera and a set of biochips containing also Bet v 1-like allergens, namely, Act c 11 from gold kiwi, Api g 1 from celery, Ara h 8 from peanut, Cor a 1 from hazel pollen, and Mal d 1 from apple, and including pBet $\mathrm{v}$ 1a, spotted for experimental purposes. Before the immobilization on the FABER biochip, pBet $\mathrm{v}$ la was coupled on nanobeads following the same procedure applied to all the other allergenic preparations. This multiplex diagnostic test allowed the detection of specific IgE to each of the allergenic preparations contained in the FABER biochip, including Bet v 1 from Escherichia coli and that from the plant, in a single run. To obtain information on shared epitopes on homologous proteins a modified single point highest inhibition achievable assay (SPHIAa) was used (Bernardi et al., 2011). IgE binding inhibition on Bet v 1-like allergens was achieved by co-incubating pBet $\mathrm{v}$ 1a with 5 sera from Bet $\mathrm{v}$ 1-allergic subjects, all having Bet v 1-specific IgE antibodies. For 
control purposes non-related IgE-positive results were used, and no inhibition was recorded (data not shown). The optimal pBet $\mathrm{v}$ la concentration for the inhibition experiments was found by preliminary experiments using a range of concentrations between $100 \mu \mathrm{g}$ and $1.25 \mu \mathrm{g} / \mathrm{ml}$ in a buffer solution. All IgE detections, either for the inhibition assay or the direct measurements, were performed in a single replicate.

Based on the current regulation on spared serum samples from the diagnostic workup, considering the venous blood sampling as part of the routine clinical practice and the observational nature of the study carried out without any action on patients themselves, a formal approval by the ethical committee or signed informed consent was not required.

\section{STATISTICS}

Statistical evaluation of protein yields and IgE distribution was made by applying the t-test for paired values and a $p<0.05$ was considered statistically significant (Graphpad Prism 5.0; Graphpad Software Inc., San Diego, CA).

\section{RESULTS}

\section{pBet v 1a Expression}

Two different transient expression systems for the expression of Bet vla in N. benthamiana plants were compared.

\section{pK7WG2 Expression}

We first conducted a time-course expression analysis for the plants transformed with the vector pK7WG2.Betv1 to determine the day of maximum expression (Figure 1). A suspension of A. tumefaciens carrying the vector was manually infiltrated into the leaves. Three leaves were sampled daily, beginning 3 until 14 dpi. Western blot analysis of the leaf extracts revealed an expression peak at 3 dpi (Figure 1). The amount of pBet v 1a at the peak expression level was evaluated by densitometric analysis and corresponded to $5.1 \pm 0.46 \mu \mathrm{g} / \mathrm{g}$ of fresh leaves weight (FLW).

\section{pGPVXGATEWAY(A) Expression}

For the infection with the Potato Virus X (PVX)-based vector, the $N$. benthamiana leaves were infiltrated with the A. tumefaciens suspension carrying pGPVXGATEWAY(A).Betv1. When the first viral symptoms appeared (10 dpi), we collected the infected leaves and analyzed them by western blot analysis. In particular, three biological replicates consisting of a collection of the leaves of three infected plants (Figure 2) were performed. On the basis of the densitometric analysis, the expression level of the recombinant protein Bet $\mathrm{v} 1$ was estimated to correspond to $51.9 \pm 2.51 \mu \mathrm{g} / \mathrm{g}$ of FLW.

\section{pBet v 1a IMAC Purification and Yields}

Small-scale purification experiments were set up, starting from plant material deriving from the two systems used for recombinant protein production in the $N$. benthamiana plants.

We independently extracted the TSP starting from $30 \mathrm{~g}$ of leaves infiltrated with pK7WG2.Betv1 and $10 \mathrm{~g}$ of leaves infected using pGPVXGATEWAY(A).Betv1. The extracts were subjected to affinity chromatography using a Ni-NTA column (Figure 3). The optimal imidazole concentration for pure protein elution was between 55 and $60 \mathrm{mM}$ (Fractions 10-13, Figure 3) and leaves infiltrated with pK7WG2.Betv1 and pGPVXGATEWAY(A).Betv1 displayed the same pattern (data not shown). In the discarded fractions 1 to 9 , we detected a protein of approximately $55 \mathrm{kDa}$ weight, which resulted to be a contaminant and not an oligomerization of $\mathrm{pBet} v$ 1a since it was not detected in the anti-FLAG ${ }^{\circledR}$ Western blot analysis. The yields of the purified recombinant protein obtained using the two systems were estimated by Western blot followed by a densitometric analysis of the protein bands recognized with the specific antibodies of three independent purified $\mathrm{pBet} v$ 1a preparations.

The average yield of purified pBet $\mathrm{v}$ la obtained from pK7WG2.Betv1 and pGPVXGATEWAY(A).Betv1 was of $3.7 \pm 0.02 \mu \mathrm{g} / \mathrm{g}$ FLW and $23.4 \pm 0.004 \mu \mathrm{g} / \mathrm{g}$ FLW, respectively (Figure 4). The stardard deviation was calculated by comparing the results of three independent purification experiments for each expression system.

\section{d.p.i.}

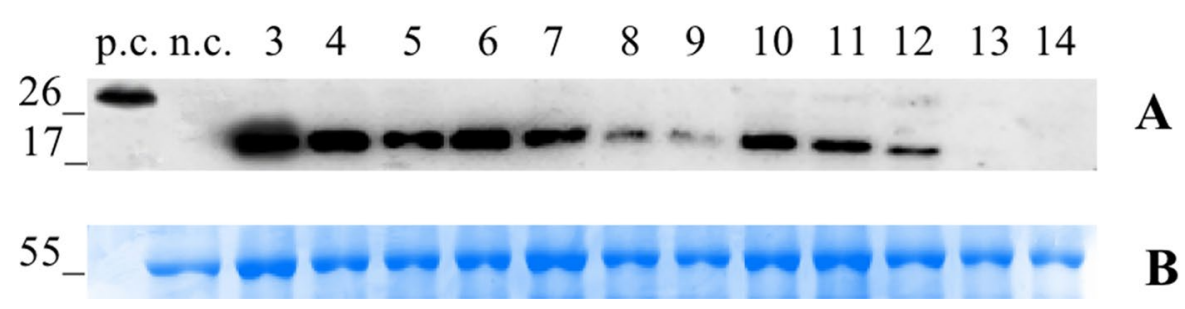

FIGURE 1 | Time-course analysis of pK $7 W G 2$. Betv1 expression of the agro-infiltrated $N$. benthamiana leaves. Western blot analysis (A) and corresponding loading controls (RuBisCO large subunit) stained with Coomassie Brilliant Blue (B), of pBet v 1a containing protein extract from leaves samples collected from 3 to 14 dpi. Each lane was loaded with $2.5 \mathrm{\mu g}$ of TSP, the western blot was probed with anti-FLAG® antibody conjugated with horseradish peroxidase. Side numbers indicate molecular mass markers in $\mathrm{kDa}$. p.c., positive control, $10 \mathrm{ng}$ of a commercially available flagged protein; n.c., negative control, extract from leaves infiltrated solely with A. tumefaciens EHA105. 


\section{replicates}

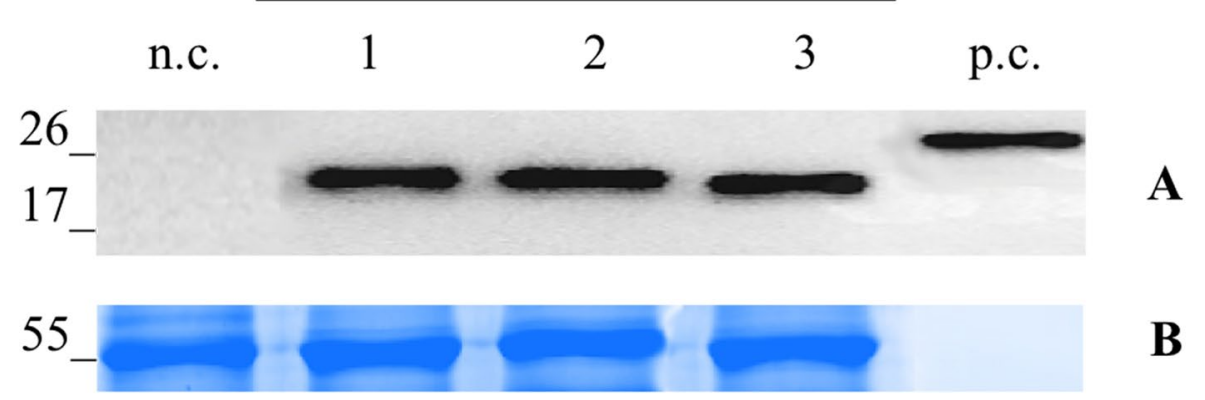

FIGURE 2 | Evaluation of extraction reproducibilty of the pG PVX GATEWAY(A).Betv1 agro-infiltrated N. benthamiana leaves. Western blot analysis (A) and corresponding loading controls (RuBisCO large subunit) stained with Coomassie Brilliant Blue (B), of pBet v 1a containing protein extract from leaves samples in three biological replicates. Each lane was loaded with $2.5 \mu \mathrm{g}$ of TSP, the western blot was probed with anti-FLAG ${ }^{\circledR}$ antibody conjugated with horseradish peroxidase. Side numbers indicate molecular mass markers in kDa. p.c., positive control, $10 \mathrm{ng}$ of acommercially available flagged protein; n.c., negative control, extract from leaves infiltrated solely with A. tumefaciens GV3101.

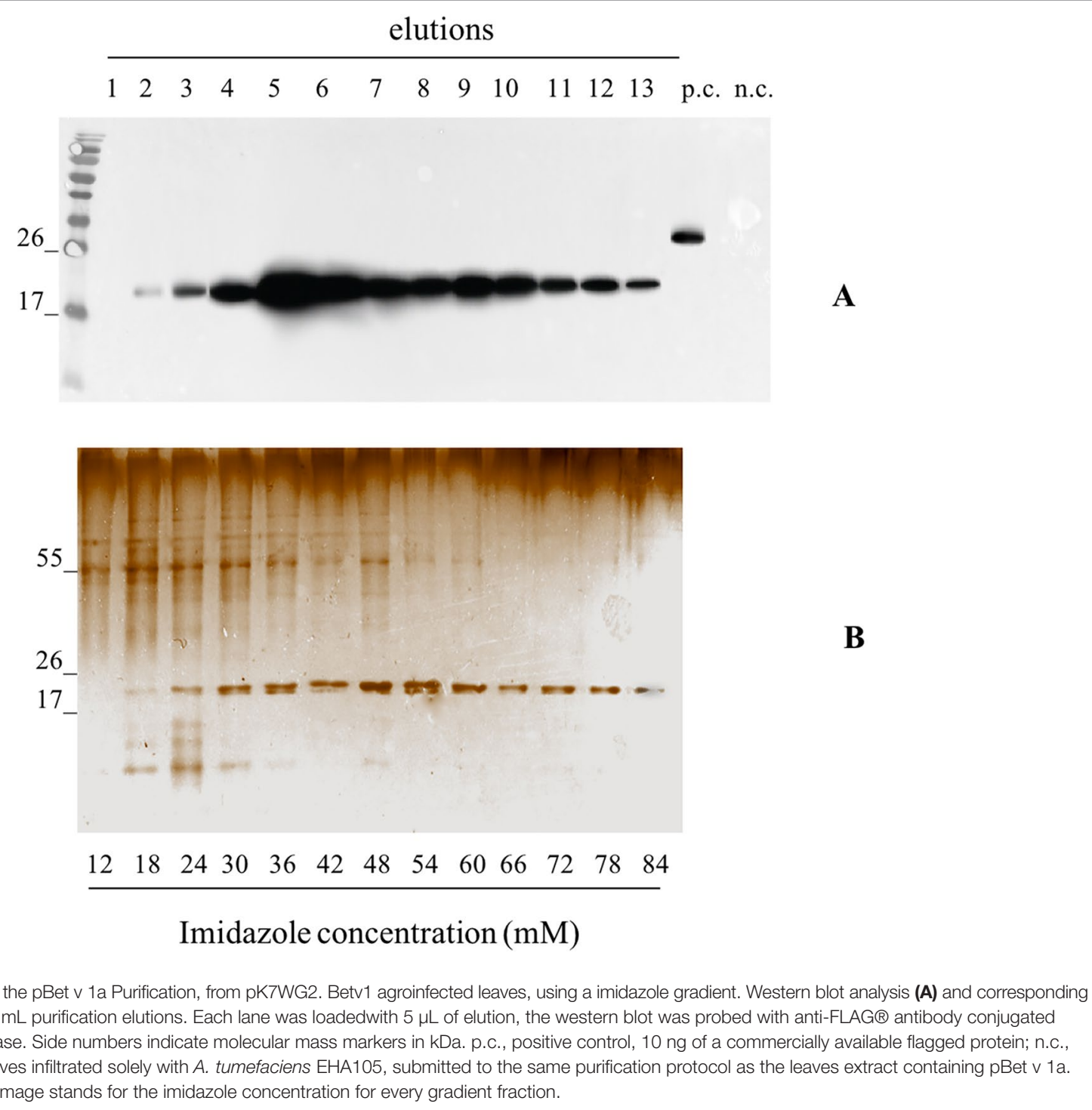




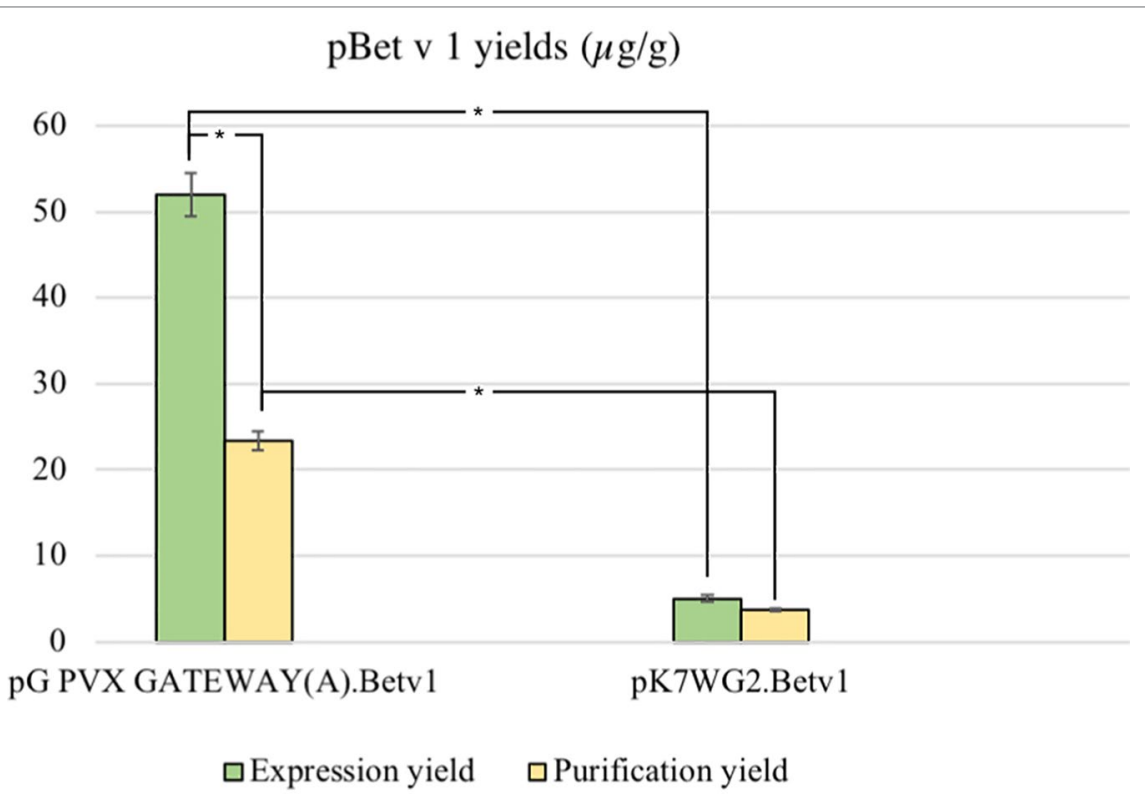

FIGURE 4 | Average yields $\mu \mathrm{g} / \mathrm{g}$ LFW of purified protein between the two expression systems. The expression and purification yields are reported, respectively, in green and yellow. The bars represent the standard deviations of three independent purification experiments, asterisks indicates the statiscal significance evaluated by t-test $(p<0.01)$.

All the susequent experiments were performed using pBet $\mathrm{v}$ 1a produced using the pGPVXGATEWAY(A).Betv1 because of the higher yields obtained.

\section{Structural Characterization of pBet v 1a Analysis of the pBet v 1a Preparation by Size Exclusion Chromatography and RP-HPLC}

Following the affinity chromatography, pBet v la was subjected to size exclusion chromatography. Figure $\mathbf{5}$ shows the elution profile of pBet $\mathrm{v}$ la compared with that of the allergen expressed in E. coli. Similar to the E. coli derived molecule, pBet v 1a was

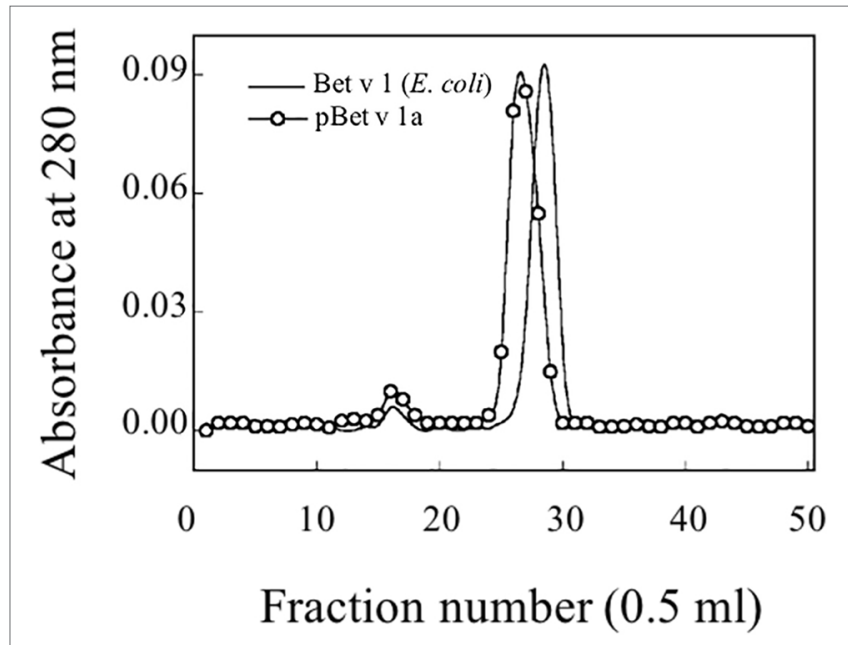

FIGURE 5 | Gel filtration profile on a Superdex 75 column of purified pBet v $1 \mathrm{a}$ and Bet $\mathrm{v} 1$ expressed in E. coli. eluted as a single peak excluding the presence of aggregated forms. As expected, pBet v 1a is eluted at a slightly lower volume due to the higher molecular weight $(19,696 \mathrm{Da})$ than that of the molecule expressed in E. coli $(17,570 \mathrm{Da})$. This is due to the presence of a tail (containing a poly-Histidine tag, a Flag-tag and a linker) of 19 amino acid residues at the $\mathrm{N}$ terminus of pBet $\mathrm{v}$ la. The fractions containing pBet $\mathrm{v}$ 1a, eluted from the gel filtration, were collected and pooled. The concentration of the pure protein obtained from size exclusion chromatography was estimated on the basis of the molar extinction coefficient, at $280 \mathrm{~nm}$.

An amount of $0.2 \mathrm{mg}$ was further analyzed by RP-HPLC (Figure 6). pBet v la was eluted as a single peak that was collected

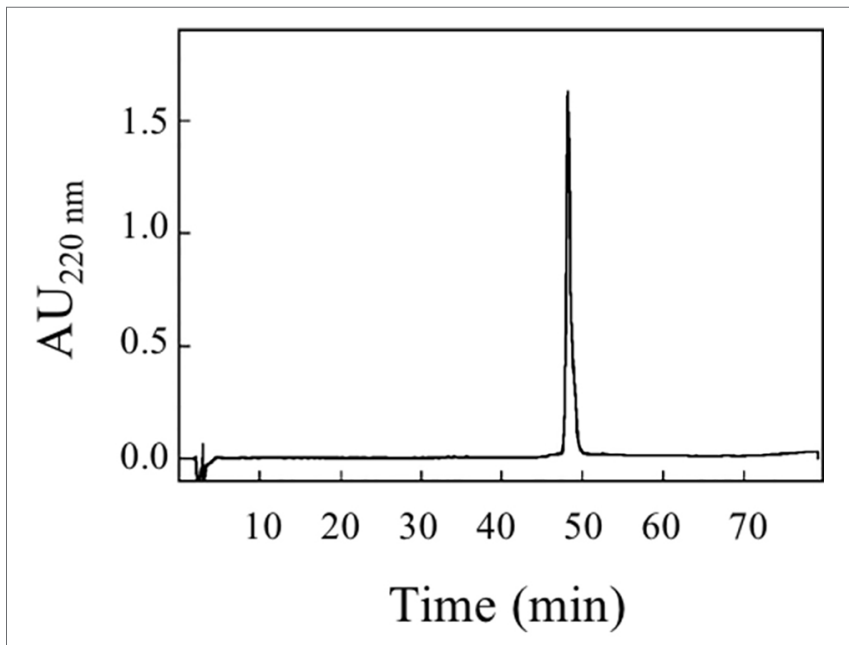

FIGURE 6 | RP-HPLC elution profile of purified pBet v 1a (200 $\mu \mathrm{g})$ 
and concentrated with a centrifugal vacuum concentrator (Savant Speedvac Plus SC110A).

\section{Assessment of pBet $v 1$ a Identity by Mass Spectrometry}

An amount of Bet $\mathrm{v}$ 1a corresponding to $5 \mu \mathrm{g}$, deriving from RP-HPLC elution, was used for the assessment of its identity using the in solution enzyme digestion method followed by shotgun proteomics (Shan et al., 2013). This procedure allowed the identification of several overlapping peptides covering $76 \%$ of the Bet v 1a protein sequence (see Figure 7). Except a peptide deriving from the trypsin enzyme used for the protein digestion, peptides belonging to any other protein contained in Uniprot database were not detected. In addition, no sequence heterogeneities were observed. Therefore, this experiment demonstrates that the plant expressed pBet $\mathrm{v}$ 1a was exactly the expected protein and that the molecule had been purified to homogeneity.

In summary, the purity of pBet $\mathrm{v}$ la eluted from size exclusion chromatography was assessed using different methods. It provided a single band on SDS-PAGE, was eluted as a single peak from RP-HPLC and was identified as a single molecule from shotgun proteomics. No evidence of the presence of posttranslational modifications was recorded.

\section{Structural Analysis by Circular Dichroism Experiments}

Figure 8 shows that the CD spectrum obtained for pBet $v$ la is different from that of the E. coli-produced allergen. The CD curve of the bacterial-derived allergen is very similar to the one reported in literature for the natural Bet v 1 (Batard et al., 2005; Bollen et al., 2007), displaying a broad minimum around $218 \mathrm{~nm}$. Conversely, the spectrum of the pBet v la shows two minima around 208 and $216 \mathrm{~nm}$, consistent with a ratio of the alfa-helix higher than that present in the structure of the molecule expressed in E. coli.

\section{Thermal Stability}

Figure 9 shows the stability of Bet v 1a at different temperatures, reported as molar ellipticity registered at $222 \mathrm{~nm}$. It can be observed that the protein maintains its structure until $80^{\circ} \mathrm{C}$ and only at $90^{\circ} \mathrm{C}$ the unfolding starts.

\section{Immunological Characterization IgE-Binding Inhibition}

As shown in Figure 10A, pBet v 1a used in solution to inhibit the IgE binding to the E.coli-produced Bet $\mathrm{v} 1$ was able to provide an inhibition of up to $100 \%$. The same was obtained for Cor a 1 and Mal d 1, whereas an almost complete inhibition was achieved with two other Bet $\mathrm{v}$ 1-like molecules from peanut and celery. These results are in line with the different degree of primary structure similarity observed by comparing the sequence of Bet $v$ la with those of the homologous allergens analyzed in this study (Figure 7). In fact, the sequence identity between Bet v 1a and the Bet v 1-like allergens Cor a 1, Mal d 1, Ara h 8 and Api g 1 is 72.5\%, 55.6\%, 46.2\% and 40.0\%, respectively. Therefore, a clear correlation between the level of IgE binding inhibition and the primary structure similarities is observed. IgE binding inhibition to kirola, Act c 11, was also observed, although the sequence identity with Bet $\mathrm{v}$ 1a is very low (12\%). However, Act c 11 (as well as Act d 11) represents a particular case since it is not included in the Bet $\mathrm{v}$ 1-like protein family. In fact, it belongs to the Major Latex Protein/Ripening Related Protein (MLP/RRP) family, but it is immunologically correlated with Bet $\mathrm{v}$ 1-like allergens with which it displays IgE co-recognition (D'Avino et al., 2011; Chruszcz et al., 2013).

\section{Direct IgE Binding}

When the pBet $\mathrm{v}$ 1a was tested for direct comparison with the one produced in E. coli on an extended number of samples, the values were differently dispersed with a mean IgE value lower for pBet v la (Figure 10B). The observation of single 304 IgE values

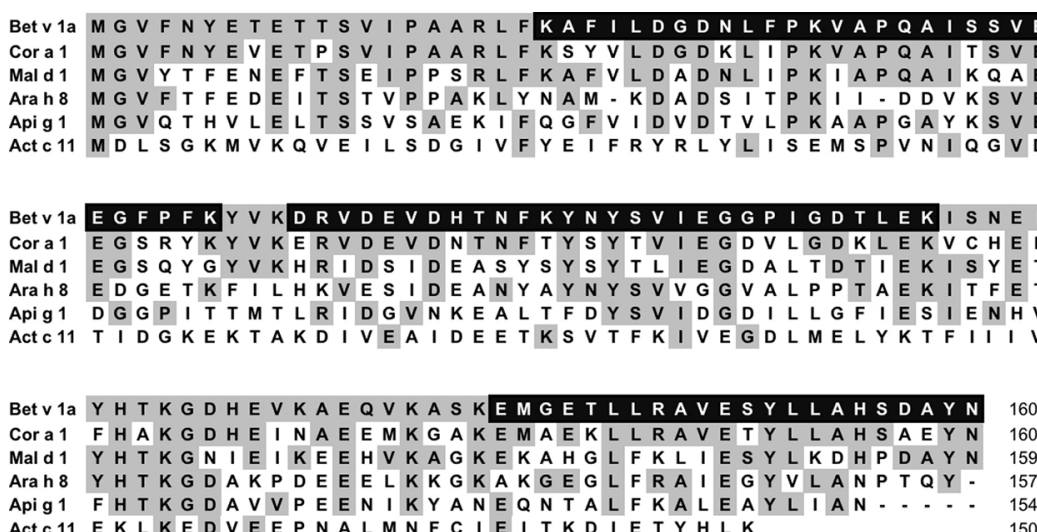

Act C11 EK L KE DVEEPN A L N F C I E I T K D I E T Y H K K

FIGURE 7 | Multiple-sequence alignment of Bet v 1a (accession number P15494) with the homologous allergens Cor a 1, Mal d 1, Ara h 8 , Api g 1 and Act c 11 having the accession number Q08407, 598 Q9SYW3, Q6VT83, P49372, AOA2R6PAW0, respectively. The residues of Bet $v 1$ a conserved in the aligned homologues are shadowed in gray. The amino acid residues experimentally identified by mass spectrometry are in white and black shadows. 


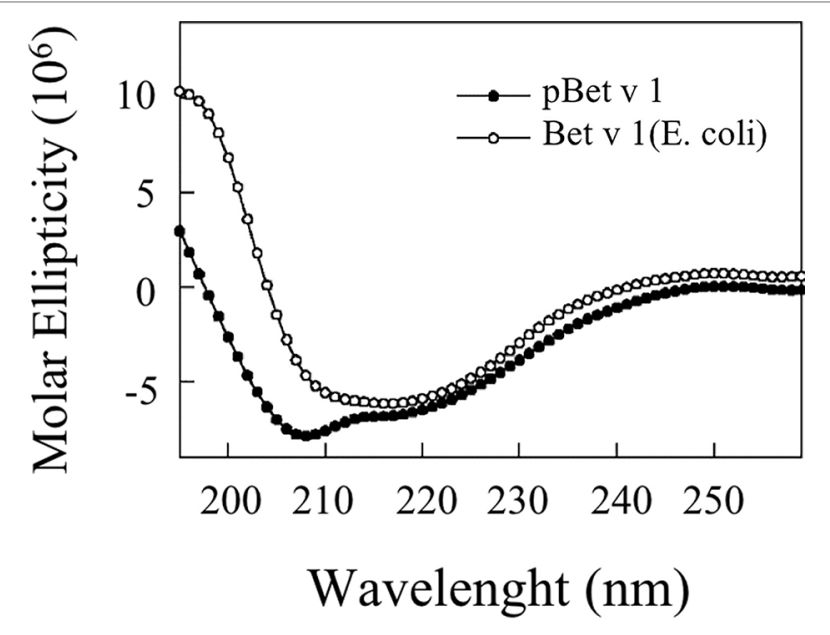

FIGURE 8 | CD spectra of plant-expressed and E. coli-expressed (commercial) Bet $\vee 1$.

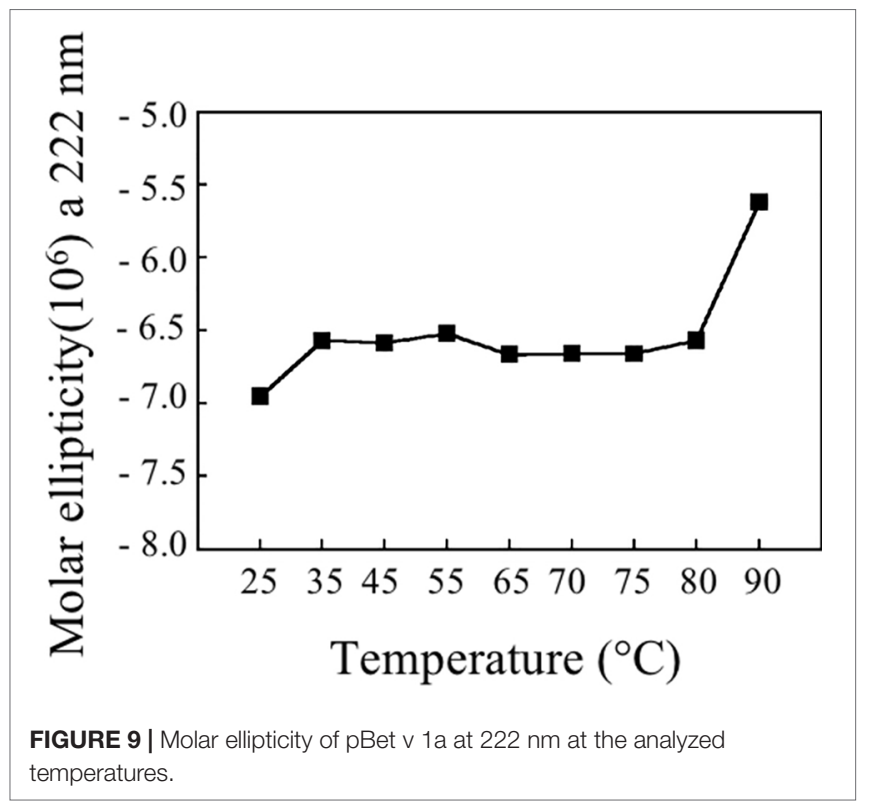

showed that some sera had almost overlapping reactivities, whereas others showed a markedly different behavior, recording a number of negative results. Comparing the IgE value distribution by using the t-test for paired values, it turned out that the series were statistically different $(p<0.0001)$.

The best performing sera belonged to the Bet v 1 IgE-positive subset having IgE reactivities also on other Bet v 1-like molecules (data not shown).

\section{DISCUSSION}

The development of tools for a precise and definitive diagnosis of allergic diseases is considered strategic for the future, both in terms of developing vaccines for immunotherapy, exploiting a companion diagnostic strategy, and of studying the genuine allergic sesitization of patients to a particular allergen source. Determining the sensitization profile of an inidvidual patient creates the opportunity to assess the individual risk of the severity of an allergic reaction and to predict the natural course (Van Gasse et al., 2015).

The progress in molecular farming exploiting different production platforms together with the current knowledge of allergen components and protein families (www.allergen. org; www.allergome.org) has boosted molecular allergy diagnosis both for food and for inhalant allergens. In this context, plant-based expression systems are considered advantageous for several reasons: (1) cost-effectiveness, (2) ease in scalability, and (3) authenticity with respect to plantderived allergens.

In this study, we have investigated and compared the performance of two different strategies for the transient expression of allergenic proteins in plants, one based on the use of a modified plant-virus and the other on a plant expression vector. The pipeline of the two different strategies is visualized in Figure 11. The virus-based mediated a higher expression level, almost ten-fold, than the plant one, as previously demonstrated for other recombinant proteins (SalazarGonzález et al., 2014). This result may be explained in terms of the higher rate of transcription of viral RNA-dependent RNA polymerase and of the natural capacity of viruses to sequester the plant apparatus for their replication in the host. However, the highest expression levels here obtained were four times (Krebitz et al., 2000) lower than those previously reported in $N$. benthamiana using a tobacco mosaic virus (TMV)-based system for the expression of the same protein. We speculate that this difference may be explained either in terms of a lower efficiency of PVX than TMV in mediating foreign protein expression or of the presence in our construct of a His-tag which may influence the recombinant protein accumulation (Pinnola et al., 2015), or even as a result of a combination of the two factors.

pBet v 1a was purified for the first time to our knowledge from $N$. benthamiana infected and infiltrated leaves using a single-step purification protocol based on affinity chromatography. The recombinant protein was purified at higher absolute yields when using the viral vector in comparison to the use of the plant-specific expression vector. However, the former was characterized by lower relative yields, probably reflecting a lower efficiency of the affinity column in capturing the antigen, this being present in higher amounts in the extracted plant soup.

Bet $\mathrm{v} 1$ is the major allergen of birch pollen. The expression of this protein was chosen for this project because it is an important and well-studied allergen for which reference information is available in the literature. In addition, the recombinant molecule from a prokaryotic expression system is available for comparative analysis. In particular, E.coli-made Bet $\mathrm{v} 1$ is already used in in vitro allergy diagnosis, and it is available as an allergen reference standard from the European 


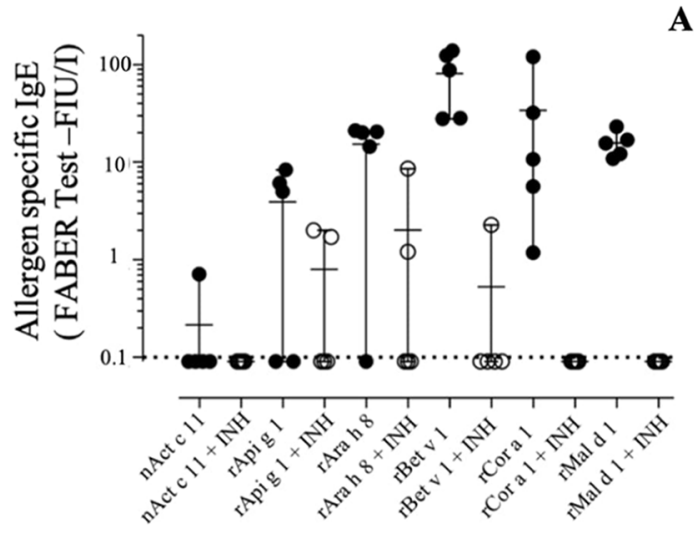

A

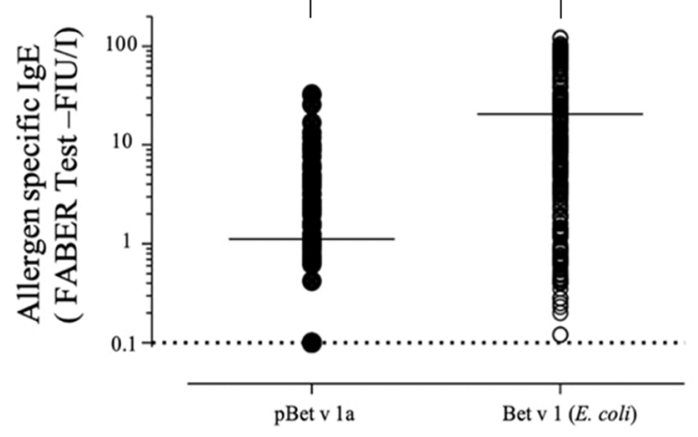

B

Allergens or Allergen plus pBet $\mathrm{v}$ 1a as Inhibitor $(\mathrm{n}=$ Natural; $\mathrm{r}=$ E. coli Recombinant; $\mathrm{INH}=\mathrm{pBet} \mathrm{v} 1 \mathrm{a})$

FIGURE 10 | (A) Specific IgE-binding inhibition using pBet v 1a on Bet v 1-like molecules on the FABER test solid phase. (B) Specific IgE direct comparative binding using 304 Bet $v 1 \mathrm{lgE}$-positive sera and statistical evaluation of IgE value distributions by using the t-test for paired values $(p<0.0001)$, displayed by the asterisk.

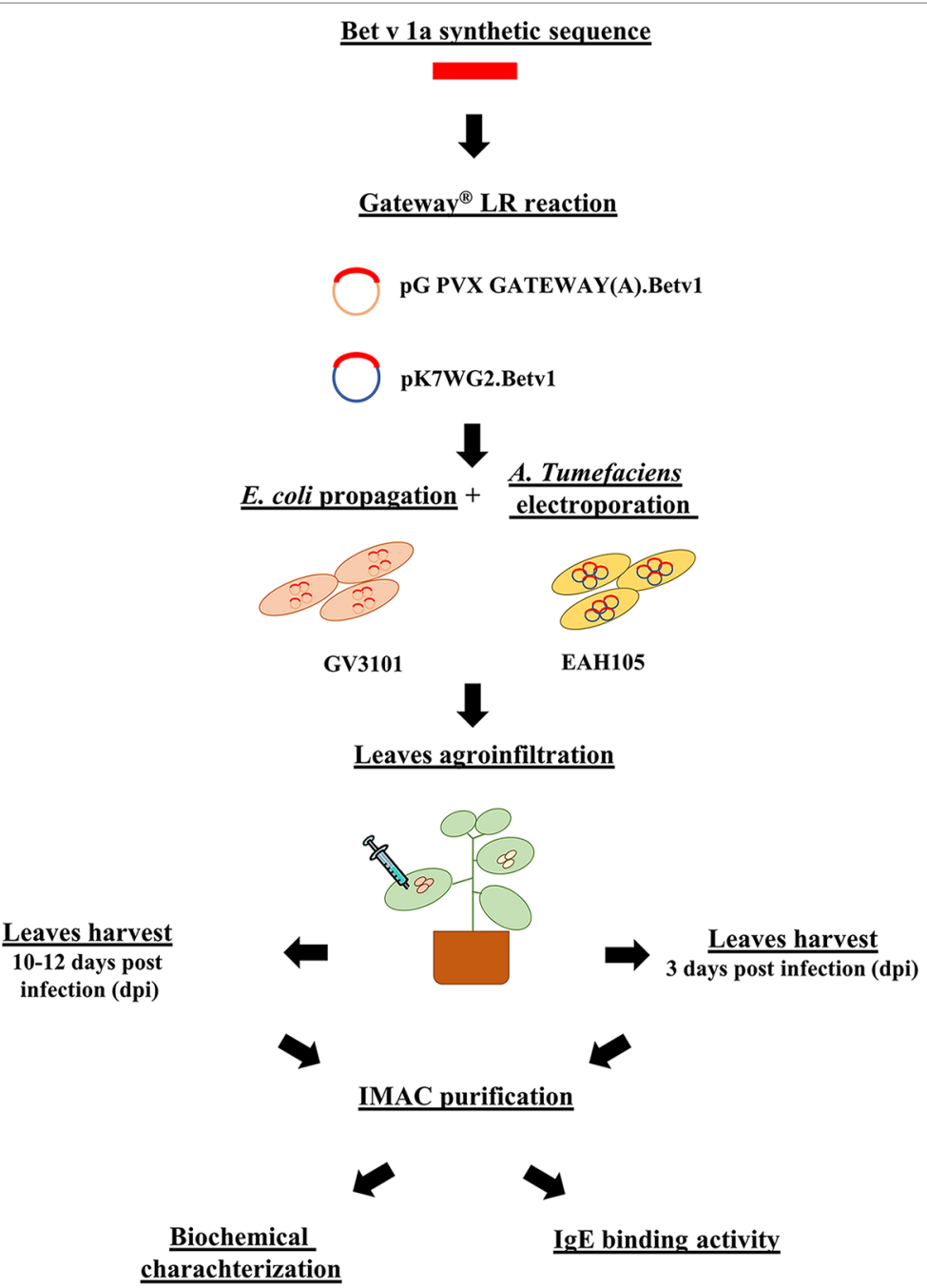

FIGURE 11 | Streamline pBet v 1a production and characterization. 
Directorate for the Quality of Medicines and Health Care, EDQM (Vieths et al., 2012).

The pBet $\mathrm{v}$ la produced in this study was recognized by the specific IgE of patients allergic to birch pollen and positive to $E$. coli-made Bet $\mathrm{v} 1$. It efficiently bound specific IgE in solution, thus inhibiting their binding with Bet $\mathrm{v} 1$ and its homologs that were immobilized on the FABER biochip. Nevertheless, in some experimental conditions, $\mathrm{pBet} \mathrm{v}$ 1a showed a different behavior with respect to the molecule expressed in the prokaryotic system. For instance, lower IgE values were often obtained for pBet $\mathrm{v}$ 1a when it was spotted on the FABER biochip and tested simultaneously with $E$. coli-made Bet v 1 . The results, obtained from an investigation of the structural features of $\mathrm{pBet}$ $\mathrm{v}$ la, suggest that its individual immunological features might be associated with a molecule conformation different from that of the prokaryotic Bet $\mathrm{v} 1$ used for comparison. In fact, CD experiments showed a higher content of the helical structure in pBet $\mathrm{v} 1 \mathrm{a}$ than in the E. coli-made protein. This is not very surprising since it is well known that the Bet $\mathrm{v} 1$ conformation is affected by various factors. Its structure is not stabilized with disulphide bridges (Radauer et al., 2008), as occurs for allergens that have a very compact structure, such as LTP and gibberellin-regulated proteins (Tuppo et al., 2014; Giangrieco et al., 2015). Although we cannot exclude the possibility that the presence of the histidine tail and flag, added at the N-terminus of pBet $\mathrm{v} 1 \mathrm{a}$, could affect its conformation. An additional factor worth considering is the Bet v 1 ligand binding (Śliwiak et al., 2016). In fact, Bet v 1 and its homologs are described as a promiscuous acceptor for a wide variety of hydrophobic ligands (Chruszcz et al., 2013; von Loetzen et al., 2013). In particular, the ligand deoxycholate has been reported to stabilize the protein conformational IgE epitopes. It has also been suggested that ligand-binding affects the allergenicity of the protein and that humans are exposed to both ligand-bound and ligand-free Bet v 1 (Asam et al., 2014). Therefore, the absence or possible presence of a specific ligand bound to pBet $\mathrm{v}$ 1a and affecting its structure and immunological behavior is something worth investigation in the near future. Sometimes, as reported also for the kiwifruit allergen Act d 5 (Bernardi et al., 2010), the availability of an allergen with different conformations is useful to have a greater number of panels of the IgE epitopes, which can be used to reveal additional sets of IgE antibodies, thus improving allergy diagnosis.

Thermostability experiments suggest that pBet $\mathrm{v}$ 1a might be more stable than the molecule expressed in E. coli and lacking the 19-residue tail present at the $\mathrm{N}$-terminus of the molecule described in this study. In fact, pBet v la is stable until $80^{\circ} \mathrm{C}$ and only at $90^{\circ} \mathrm{C}$ starts to loose its secondary structure elements. In contrast, a melting point of $66^{\circ} \mathrm{C}$ was reported for Bet $\mathrm{v} 1$ expressed in E. coli (Himly et al., 2009). Further studies are necessary to understand whether the 19-residue tail of pBet v 1a has a stabilizing effect on the structure of this molecule.

In conclusion, the results of this study highlight the valuable performance of the plant-based expression system tested with the major birch allergen Bet $\mathrm{v}$ 1a. This represents a feasible platform for the production of the major allergen of birch, being characterized by high yields and a simple, one-step, protocol for recombinant protein purification. We, therefore, suggest that this platform may be used succesfully also for other recombinant allergens whose production is not feasible in bacterial cells. In addition, this technique could be exploited for the development of immunotherapeutic strategies, also considering their potential for oral administered vaccines. In fact, as recently demonstrated for other allergens (Fukuda et al., 2018), the administration to humans of molecules deriving from edible plant organs can be a safer approach than using those obtained from non-edible sources.

\section{AUTHOR CONTRIBUTIONS}

MS performed the transient pBet $\mathrm{v}$ 1a expression in plants, its characterization and purification. IG performed the structural characterization of the expressed protein by $\mathrm{CD}$, size exclusion chromatography, RP-HPLC and thermal stability experiments and conjugated pBet $\mathrm{v}$ la to the nanobeads for the FABER test. MAC contributed in manuscript writing. RZ supervised the upstream and downstream process set-up. MP contributed in the experimental design. CR, MC, and AM designed, performed, and supervised all the immunological experiments. LA designed the experiments, coordinated all the experimental activities, and wrote the manuscript.

\section{ACKNOWLEDGMENTS}

We acknowledge the Department of Biotechnology of the University of Verona for funding the bursary of Mattia Santoni. We thank Dr Gabriella Pocsfalvi and Dr Immacolata Fiume of the the mass spectrometry and proteomics facility, at IBBR (CNR), for the mass spectrometry experiments and Dr. Jon Cole for the scientific English revision of the manuscript.

\section{REFERENCES}

Alessandri, C., Ferrara, R., Bernardi, M. L., Zennaro, D., Tuppo, L., and Giangrieco, I. (2017). Diagnosing allergic sensitizations in the third millennium: why clinicians should know allergen molecule structures. Clin. Transl. Allergy 7, 21. doi: 10.1186/s13601-017-0158-7

Asam, C., Batista, A. L., Moraes, A. H., de Paula, V. S., Almeida, F. C. L., Aglas, L., et al. (2014). Bet v 1-a Trojan horse for small ligands boosting allergic sensitization? Clin. Exp. Allergy 44 (8), 1083-1093. doi: 10.1111/cea.12361

Avesani, L., Marconi, G., and Morandini, F. (2007). Stability of Potato virus X expression vectors is related to insert size: implications for replication models and risk assessment. 16 (5), 587-597. doi: 10.1007/s11248-006-9051-1

Batard, T., Didierlaurent, A., Chabre, H., Mothes, N., Bussières, L., Bohle, B., et al. (2005). Characterization of wild-type recombinant Bet v la as a candidate vaccine against birch pollen allergy. Int. Arch. Allergy Immunol. 136(3), 239-249. doi: 10.1159/000083950

Bernardi M. L., Giangrieco I., Camardella L., Ferrara R., Palazzo P., Panico M. R., et al. (2011). Allergenic lipid transfer proteins from plant-derived foods do not immunologically and clinically behave homogeneously: the kiwifruit LTP as a model. PLoS One 6, e27856. doi: 10.1371/journal.pone.0027856.

Bernardi, M. L., Picone, D., Tuppo, L., Giangrieco, I., Petrella, G., Palazzo, P., et al. (2010). Physico-chemical features of the environment affect the protein conformation and the immunoglobulin E reactivity of kiwellin (Act d 5). Clin. Exp. Allergy. doi: 10.1111/j.1365-2222.2010.03603.x 
Bollen, M. A., Garcia, A., Cordewener, J. H. G., Wichers, H. J., Helsper, J. P. F. G., Savelkoul, H. F. J., et al. (2007). Purification and characterization of natural Bet $\mathrm{v} 1$ from birch pollen and related allergens from carrot and celery. Mol. Nutr. Food Res. doi: 10.1002/mnfr.200700119

Bortesi, L., Rossato, M., Schuster, F., Raven, N., Stadlmann, J., Avesani, L., et al. (2009). Viral and murine interleukin-10 are correctly processed and retain their biological activity when produced in tobacco. BMC Biotechnol. 9, 9-22. doi: 10.1186/1472-6750-9-22

Chruszcz, M., Ciardiello, M. A., Osinski, T., Majorek, K. A., Giangrieco, I., Font, J., et al. (2013). Structural and bioinformatic analysis of the kiwifruit allergen Act d 11, a member of the family of ripening-related proteins. Mol. Immunol. 56, 794-803. doi: 10.1016/j.molimm.2013.07.004

Ciardiello, M. A., Giangrieco, I., Tuppo, L., Tamburrini, M., Buccheri, M., Palazzo, P., et al. (2009). Influence of the natural ripening stage, cold storage, and ethylene treatment on the protein and ige-binding profiles of green and gold Kiwi fruit extracts. J. Agric. Food Chem. 57 (4), 1565-1571. doi: 10.1021/jf802966n

Ciardiello, M. A., Tamburrini, M., Liso, M., Crescenzo, R., Rafaiani, C., and Mari, A. (2013). Food allergen profiling: a big challenge. Food Res. Int. 54 (1), $1033-$ 1041. doi: 10.1016/j.foodres.2013.03.013

D’Avino, R., Bernardi, M. L., Wallner, M., Palazzo, P., Camardella, L., Tuppo, L., et al. (2011). Kiwifruit Act d 11 is the first member of the ripening-related protein family identified as an allergen. Allergy Eur. J. Allergy Clin. Immunol. 66, 870877. doi: 10.1111/j.1398-9995.2011.02555.x

Fukuda, K., Ishida, W., Harada, Y., Wakasa, Y., Takagi, H., Takaiwa, F., et al. (2018). Efficacy of oral immunotherapy with a rice-based edible vaccine containing hypoallergenic Japanese cedar pollen allergens for treatment of established allergic conjunctivitis in mice. Allergol. Int. 67 (1), 119-123. doi: 10.1016/j. alit.2017.06.006

Gecchele, E., Merlin, M., Brozzetti, A., Falorni, A., Pezzotti, M., and Avesani, L. (2015). A Comparative analysis of recombinant protein expression in different biofactories: bacteria, insect cells and plant systems. J. Vis. Exp. 97. doi: $10.3791 / 52459$

Giangrieco, I., Alessandri, C., Rafaiani, C., Santoro, M., Zuzzi, S., Tuppo, L., et al. (2015). Structural features, IgE binding and preliminary clinical findings of the $7 \mathrm{kDa}$ lipid transfer protein from tomato seeds. Mol. Immunol. 66 (2), 154-163. doi: 10.1016/j.molimm.2015.02.025

Giangrieco, I., Rafaiani, C., Liso, M., Palazzo, P., Pomponi, D., Tuppo, L., et al. (2012). Allergens in allergy diagnosis: a glimpse at emerging new concepts and methodologies. Transl. Med. @UniSa 4, 27-33.

Himly, M., Nony, E., Chabre, H., Van Overtvelt, L., Neubauer, A., Van Ree, R., et al. (2009). Standardization of allergen products: 1 . Detailed characterization of GMP-produced recombinant Bet v 1.0101 as biological reference preparation. Allergy Eur. J. Allergy Clin. Immunol. 64, 1038-1045. doi: 10.1111/j.1398-9995.2009.01957.x

Jarolim, E., Rumpold, H., Endler, A. T., Ebner, H., Breitenbach, M., Scheiner, O., et al. (1989). IgE and IgG antibodies of patients with allergy to birch pollen as tools to define the allergen profile of Betula verrucosa*. Allergy 44 (6), 385-395 doi: 10.1111/j.1398-9995.1989.tb04169.x

Karimi, M., Inzé, D., and Depicker, A. (2002). GATEWAYTM vectors for Agrobacterium-mediated plant transformation. Trends Plant Sci. 7, 193-195. doi: 10.1016/S1360-1385(02)02251-3

Krebitz, M., Wiedermann, U., Essl, D., Steinkellner, H., Wagner, B., Turpen, T. H., et al. (2000). Rapid production of the major birch pollen allergen Bet v 1 in Nicotiana benthamiana plants and its immunological in vitro and in vivo characterization. FASEB J. 14, 1279-1288. doi: 10.1096/fasebj.14.10.1279

Lau, S., Matricardi, P. M., Wahn, U., Lee, Y. A., and K., T. (2018). Allergy and atopy from infancy to adulthood: messages from the German birth cohort MAS. Ann. Allergy Asthma Immunol. 122 (1), 25-32. doi: 10.1016/j.anai.2018.05.012

Mortz, E., Krogh, T. N., Vorum, H., and Görg, A. (2001). Improved silver staining protocols for high sensitivity protein identification using matrix-assisted laser desorption/ionization-time of flight analysis. Proteomics 1, 1359-1363. doi: 10.1002/1615-9861(200111)1:11<1359::AID-PROT1359>3.3.CO;2-H

Niederberger, V., Pauli, G., Grönlund, H., Fröschl, R., Rumpold, H., Kraft, D., et al. (1998). Recombinant birch pollen allergens (rBet v 1 and rBet v 2) contain most of the IgE epitopes present in birch, alder, hornbeam, hazel, and oak pollen: a quantitative IgE inhibition study with sera from different population. J. Allergy Clin. Immunol. 102, 579-591. doi: 10.1016/ S0091-6749(98)70273-8

Offermann, L. R., Giangrieco, I., Perdue, M. L., Zuzzi, S., Santoro, M., Tamburrini, M., et al. (2015). Elusive structural, functional, and immunological features of Act d 5, the green kiwifruit kiwellin. J. Agric. Food Chem. 63, 6567-6576. doi: 10.1021/acs.jafc.5b02159

Pasquariello, M. S., Palazzo, P., Tuppo, L., Liso, M., Petriccione, M., Rega, P., et al. (2012). Analysis of the potential allergenicity of traditional apple cultivars by Multiplex Biochip-Based Immunoassay. Food Chem. 135 (1), 219-227 doi: 10.1016/j.foodchem.2012.04.075

Pinnola, A., Ghin, L., Gecchele, E., Merlin, M., Alboresi, A., Avesani, L., et al. (2015). Heterologous expression of moss light-harvesting complex stressrelated 1(LHCSR1), the chlorophyll A-xanthophyll pigment-protein complex catalyzing non-photochemical quenching, in Nicotiana sp. J. Biol. Chem. doi: 10.1074/jbc.M115.668798

Radauer, C., Bublin, M., Wagner, S., Mari, A., and Breiteneder, H. (2008). Allergens are distributed into few protein families and possess a restricted number of biochemical functions. J. Allergy Clin. Immunol. 121 (4), 847-852. e7. doi: 10.1016/j.jaci.2008.01.025

Sampson, H. A. (2004). Update on food allergy. J. Allergy Clin. Immunol. 113 (5), 805-819. doi: 10.1016/j.jaci.2004.03.014

Salazar-González, J. A., Rosales-Mendoza, S., and Bañuelos-Hernández, B. (2014). "Viral vector-based expression strategies," in Genetically engineered plants as a source of vaccines against wide spread diseases: an integrated view. 43-60. doi: 10.1007/978-1-4939-0850-9 3

Shan, B., Yates, J. R., Baek, M.-C., Zhang, Y., and Fonslow, B. R. (2013). Protein analysis by shotgun/bottom-up proteomics. Chem. Rev. 113, 2343-2394. doi: $10.1021 / \mathrm{cr} 3003533$

Śliwiak, J., Dolot, R., Michalska, K., Szpotkowski, K., Bujacz, G., Sikorski, M., et al. (2016). Crystallographic and CD probing of ligand-induced conformational changes in a plant PR-10 protein. J. Struct. Biol. 193 (1) 55-66. doi: 10.1016/j.jsb.2015.11.008

Tuppo L., Giangrieco I., Alessandri C., Ricciardi T., Rafaiani C., Ciancamerla M., et al. (2018). Pomegranate chitinase III: Identification of a new allergen and analysis of sensitization patterns to chitinases. Mol. Immunol. 103, 89-95. doi: 10.1016/j.molimm.2018.09.009

Tuppo, L., Spadaccini, R., Alessandri, C., Wienk, H., Boelens, R., Giangrieco, I., et al. (2014). Structure, stability, and IgE binding of the peach allergen peamaclein (Pru p 7). Biopolymers 102 (5), 416-425. doi: 10.1002/bip.22530

Van Gasse, A. L., Mangodt, E. A., Faber, M., Sabato, V., Bridts, C. H., and Ebo, D. G. (2015). Molecular allergy diagnosis: status anno 2015. Clin. Chim. Acta 444 (15), 54-61. doi: 10.1016/j.cca.2015.02.012

Vieths, S., Barber, D., Chapman, M., Costanzo, A., Daas, A., Fiebig, H., et al. (2012). Establishment of recombinant major allergens Bet v 1 and $\mathrm{Phl} \mathrm{p} 5 \mathrm{a}$ as $\mathrm{Ph}$. Eur. reference standards and validation of ELISA methods for their measurement. Results from feasibility studies. Pharmeuropa Biosci. Notes 2012, 118-134.

von Loetzen, C. S., Hoffmann, T., Hartl, M., Schweimer, K., Schwab, W., Rösch, P., et al. (2013). "Identification of the natural ligand of Bet v 1", in 5th International Symposium on Molecular Allergology (ISMA 2013), Vienna, Austria, 6-7 December 2013

Conflict of Interest: The authors declare that the research was conducted in the absence of any commercial or financial relationships that could be construed as a potential conflict of interest.

Copyright (C) 2019 Santoni, Ciardiello, Zampieri, Pezzotti, Giangrieco, Rafaiani, Ciancamerla, Mari and Avesani. This is an open-access article distributed under the terms of the Creative Commons Attribution License (CC BY). The use, distribution or reproduction in other forums is permitted, provided the original author(s) and the copyright owner(s) are credited and that the original publication in this journal is cited, in accordance with accepted academic practice. No use, distribution or reproduction is permitted which does not comply with these terms. 